\title{
Urologist participation in Medicare Shared Savings Program Accountable Care Organizations (ACOs)
}

\author{
Scott R. Hawken, MS, Lindsey A. Herrel, MD, MS, Chandy Ellimoottil, MD, MS, Zaojun Ye, \\ MS, Brent K. Hollenbeck, MD, MS, and David C. Miller, MD, MPH \\ Department of Urology, University of Michigan, Ann Arbor, MI
}

\section{Structured Abstract}

Objectives-To understand the current role of urologists in Medicare Shared Savings Program (MSSP) Accountable Care Organizations (ACOs) and the organizational characteristics of ACOs with participating urologists.

\begin{abstract}
Methods-Using 2012-2013 Medicare data and the National Provider Identifier (NPI) Database, we classified each urologist in the U.S. and Puerto Rico as either an MSSP ACO-participant or non-participating provider. We then examined the distribution of ACO-participating urologists across the U.S. and among the first 220 MSSP ACOs. We also compared the characteristics of ACOs with and without participating urologists.

Results-Among 11,084 identified urologists, 1,118 (10\%) were MSSP ACO-participants. ACOparticipating urologists practiced more frequently in the Northeast and Midwest $(\mathrm{p}<0.001)$, and were more commonly female ( $10 \%$ vs. $8 \%, \mathrm{p}=0.003$ ). At an organizational level, only 110 (50\%) of the initial MSSP ACOs included at least one urologist; among this group, the number of participating urologists ranged from 1 to 55 . ACOs with one or more participating urologist were larger organizations, with respect to both the number of assigned beneficiaries and the number of providers per 1,000 beneficiaries ( $\mathrm{p}<0.001$ for each comparison). The patient populations served by ACOs with and without urologists were similar ( $>0.05$ for each comparison).
\end{abstract}

Conclusions-A modest percentage of urologists participate in MSSP ACOs, although many of these organizations still lack any formal involvement by urological surgeons. Without such participation, improving the coordination, quality, and cost of urologic care for Medicare beneficiaries may be more challenging.

\section{Keywords}

Accountable Care Organizations; Urology; Medicare; Health Policy; Workforce

Corresponding Author: David C. Miller, MD, MPH, Associate Professor, Department of Urology, University of Michigan, NCRC Building 16, Room 108E, 2800 Plymouth Road, Ann Arbor, MI 48109-2800, Phone: (734) 936-0054, Fax: (734) 232-2400 dcmiller@med.umich.edu.

Publisher's Disclaimer: This is a PDF file of an unedited manuscript that has been accepted for publication. As a service to our customers we are providing this early version of the manuscript. The manuscript will undergo copyediting, typesetting, and review of the resulting proof before it is published in its final citable form. Please note that during the production process errors may be discovered which could affect the content, and all legal disclaimers that apply to the journal pertain. 


\section{Introduction}

Because urological conditions are most prevalent in older adults, ${ }^{1}$ many predict that as the Medicare-eligible population continues to grow ${ }^{2}$ there will be a concurrent increase in the demand for urological services. ${ }^{3}$ At the same time, surgical care is a major contributor to health care costs, ${ }^{4}$ and older adults tend to utilize these services at a greater rate than the general population. ${ }^{5}$ Accordingly, there is reason to believe that the already high cost of healthcare in the U.S. may continue to increase. ${ }^{6}$

In this setting, the Centers for Medicare and Medicaid Services (CMS) have set goals to transition rapidly over the next several years from fee-for-service to alternative payment models. ${ }^{7}$ One of the most prominent alternative payment models is the Medicare Shared Savings Program (MSSP) Accountable Care Organizations (ACOs) program. The policies establishing MSSP ACOs structured their formation around physicians that deliver primary care services, ${ }^{8}$ however, if these organizations aim to improve the quality and cost of urologic care, it seems likely that they will need to engage the specialty. Still, the extent to which urologists actually participate in MSSP ACOs and whether their participation varies across regions or organizations is largely unknown.

In order to understand the role of urologists in MSSP ACOs, we examined the geographic distribution of ACO-participating urologists from 2012 to 2013, the first implementation year for the MSSP model. In addition, we investigated whether the degree of urologist participation varies across ACOs, and if there are structural attributes of ACOs that correlate with urologist participation. Because the rules governing MSSP ACO formation do not require specialist involvement, we hypothesized that urologist participation would be relatively low overall, and highly variable across organizations.

\section{Material and Methods}

\section{Identification of ACO-Participating Urologists}

We used three datasets to perform our analyses. First, we utilized the Shared Savings Program Accountable Care Organizations (ACO) Provider-Level Research Identifiable File (RIF) from CMS to identify all formal participants from April 2012 through December 2013 for each of the first 220 MSSP ACOs. ${ }^{9}$ This file contains a record for each individual MSSP ACO-participating provider, including their respective National Provider Identifier (NPI), CMS Specialty Code, and the ACO in which they participate. Using the Provider-Level RIF, we classified each unique NPI associated with the CMS Specialty Code for urologists ("34") as an ACO-participating urologist. ${ }^{10}$

\section{Identification of Non-Participating Urologists}

Second, using the National Provider Identifier (NPI) database, ${ }^{11,12}$ we ascertained the unique NPI and self-reported characteristics of all active physicians that identified as a urologist (primary provider taxonomy codes "208800000X" and "2088P0231X") in the U.S. and Puerto Rico. ${ }^{13}$ Next, in order to classify these urologists as MSSP ACO-participants versus non-participants, we linked the NPI database to the Provider-Level RIF using the NPI as a linkage variable. All urologists present in both the Provider-Level RIF and NPI database 
were classified as MSSP ACO-participating providers, and those that were absent from the Provider-Level RIF but present in the NPI database were classified as non-participants.

\section{Identification of ACOs}

Third, to examine the characteristics of ACOs with and without participating urologists, we utilized the Shared Savings Program ACO Public Use File (PUF) ${ }^{14}$ released by CMS. This file contains summarized provider and beneficiary information at the ACO-level for each of the first 220 MSSP ACOs during the first year of the program, corresponding with the time period for the Provider-Level file. We linked ACO-participating urologists from the Provider-Level RIF to their respective ACO in the PUF using a unique identifier present in both files. We then used this file to compare the organizational characteristics of ACOs with and without participating urologists.

\section{Distribution of ACO-participating Urologists}

Using urologists' self-reported practice location from the NPI database, we mapped the geographic distribution of urologist participation in MSSP ACOs. ${ }^{15}$ Next, we calculated the number of ACO-participating urologists per 10,000 beneficiaries in each of the 220 MSSP ACOs.

\section{Statistical Analysis}

We then used chi square tests to compare the distribution of ACO-participating urologists across U.S. Census regions, as well as the distribution of provider demographics according to ACO participation status.

Finally, we used Student's t-tests and non-parametric Wilcoxon rank sum tests, where appropriate, to analyze ACO-level differences among organizations with and without participating urologists. All statistical testing was performed at the 5\% significance level using SAS v9.3 (SAS Institute Inc., Cary, NC, USA). This study was deemed exempt from review by the University of Michigan institutional review board (HUM00106160).

\section{Results}

Among the 11,084 urologists identified nationwide, 1,118 (10\%) were classified as ACOparticipating urologists; the remaining 9,966 (90\%) were classified as non-participants (Electronic Supplementary Material).

Figure 1 presents the geographic distribution of urologists participating in MSSP ACOs across the U.S. There is significant variation in the distribution of participating urologists across census regions ( $<<0.001$, Table 1), with those in the Northeast and Midwest more commonly participating in an MSSP ACO. In addition, $10 \%$ of ACO-participating urologists are female, compared to only $8 \%$ of non-participating urologists $(\mathrm{p}=0.003$, Table 1$)$.

Among the 220 MSSP ACOs, 110 (50\%) did not have any formally participating urologists. For the remaining 110 organizations, there was wide variation in the degree of urologists' involvement, as measured by both the total number of participating urologists and the number of participating urologists per 10,000 beneficiaries (Figure 2). The ACO with the 
greatest involvement had a total of 55 formally participating urologists, corresponding to 61 urologists per 10,000 beneficiaries. There were 21 organizations with only one participating urologist, the largest of which had less than one urologist per 10,000 beneficiaries.

ACOs that included urologists had both a greater number of assigned Medicare beneficiaries, and more total providers per 1,000 beneficiaries $(\mathrm{p}<0.001$ for each comparison, Table 2). Patient characteristics were similar for ACOs with and without urologist participation ( $p>0.05$ for each comparison, Table 2).

\section{Comment}

We found that only $10 \%$ of all urologists nationwide formally participated in an MSSP ACO from 2012 to 2013. Moreover, fully half of all MSSP ACOs did not have any participating urologists. Among the organizations that did include urologists, there was wide variation in the degree of participation as measured both by the total number of urologists and the number of urologists per beneficiary.

Previous investigators have demonstrated that early Medicare ACOs devoted relatively little attention to surgical care. ${ }^{16}$ In this context, our finding that half of MSSP ACOs do not have any participating urologists may not be surprising. Moreover, others have suggested that subspecialists may be most prevalent in larger ACOs and/or ACOs formed from existing integrated delivery systems. ${ }^{17,18}$ Our findings generally support this hypothesis with empirical evidence demonstrating that MSSP ACOs with participating urologists are larger organizations, in terms of both beneficiaries and providers, than ACOs not formally involving urologists.

In addition, MSSP ACOs with the greatest number of urologists include several wellrecognized academic medical centers and integrated delivery systems such as the University of California, Los Angeles, the Physician Organization of Michigan ACO (which includes the University of Michigan Health System), and the Lahey Clinical Performance ACO (which includes Lahey Health). Given the structural diversity of the first 220 MSSP ACOs, future work examining whether organizational attributes are associated with meaningful improvements in urological care delivery will be highly informative.

This study has several limitations. First, the accuracy of the NPI database depends upon physicians' self-reported information that is voluntary in nature. ${ }^{12}$ Although all physicians in the U.S. are required to apply for an NPI, some may inaccurately report their personal information (e.g. specialty and practice location) and/or neglect to update this information. Notably, the total number of practicing urologists reported in the 2014 AUA Census is generally comparable to the number identified in our study, at 11,703 and 11,084 respectively, with females representing approximately $8 \%$ of both populations. ${ }^{19}$ Although it remains unclear how reliably urologists update their practice location, ${ }^{12}$ the distribution of urologists across the U.S. in our study was also comparable to results from the recent AUA Census. ${ }^{19}$ Second, it is likely that some urologists interact with MSSP ACOs without formally participating, for instance through employment by a participating hospital. Our analyses cannot identify and account for these more informal relationships. Nonetheless, there were relatively few hospital-based ACOs in our study, and it's possible that urologists 
working in these facilities may not even be aware of this ACO status if the decision to participate was primarily driven by the hospital. Finally, our data on ACO participation was limited to the first performance period for MSSP ACOs, from April 2012 through December 2013, and did not include information on changes in urologist participation in over time, Medicare's Pioneer ACOs, or commercial ACOs. Nevertheless, the MSSP ACO program is the largest federal program, and includes participants from across the country. ${ }^{20}$ Given the early nature of these data, it will be important to assess how the maturation of ACO programs, and other alternative payment models, impact urologist participation in ACOs.

These limitations notwithstanding, our findings have implications for policymakers, urologists, and patients. ACOs with few or no participating urologists may have difficulty controlling the quality and costs of urologic care, as beneficiaries seeking care outside of an ACO's network of providers may diminish organizational accountability. ${ }^{21}$ If evidence to this effect emerges, policymakers may consider modifications to ACO formation that incentivize and/or require formal participation by urologists and other specialists. For urologists, as the influence of alternative payment models continues to grow, it will be necessary to consider the potential impact of ACO participation on referral patterns and opportunities to participate in efforts aimed at improving the quality and cost-efficiency of urological care. ${ }^{16,22}$ For patients, the variation in urologist participation across ACOs may translate into differences in quality of care coordination for various urological conditions.

Moving forward, continued assessment of the effect of urologists' ACO participation on these issues will be critical, and defining the impact of ACO participation on urology practices will be essential to understanding the implications of this model for the urology workforce.

\section{Conclusions}

Although a modest percentage of urologists participate in MSSP ACOs, half of these organizations still lack any formal involvement by urological surgeons. In the absence of urologist participation, improving the coordination, quality, and cost of urological care for Medicare beneficiaries may be more challenging. Future studies examining the ability of ACO participating urologists to improve care delivery will inform the impact this model on urological care and the implications for the urology workforce.

\section{Supplementary Material}

Refer to Web version on PubMed Central for supplementary material.

\section{Acknowledgments}

This work was supported by funding from the National Cancer Institute (1-RO1-CA-174768 to DCM), the American Cancer Society (RSGI-13-323-01-CPHPS to BKH), the National Institute on Aging (R01-AG048071 to BKH), the Agency for Healthcare Research and Quality (1F32HS024193-01 to CE), the National Institute of Diabetes and Digestive and Kidney Diseases (5T32DK007782 to LAH), and the National Center for Advancing Translational Sciences (2UL1TR000433-06 to SRH). 


\section{References}

1. Miller DC, Saigal CS, Litwin MS. The Demographic Burden of Urologic Diseases in America. Urol Clin North Am. 2009; 36(1):11-27. [PubMed: 19038632]

2. U.S. Census Bureau. [Accessed August 18, 2015] Projections of the Size and Composition of the US Population: 2014 to 2060. Available at: https://www.census.gov/content/dam/Census/library/ publications/2015/demo/p25-1143.pdf

3. Pruthi RS, Neuwahl S, Nielsen ME, Fraher E. Recent Trends in the Urology Workforce in the United States. Urology. 2013; 82(5):987-994. [PubMed: 24055244]

4. Laugesen MJ, Glied SA. Higher fees paid to US physicians drive higher spending for physician services compared to other countries. Health Aff. 2011; 30(9):1647-1656.

5. Williams TE, Satiani B, Thomas A, Ellison EC. The impending shortage and the estimated cost of training the future surgical workforce. Ann Surg. 2009; 250(4):590-597. [PubMed: 19730238]

6. Centers for Medicare and Medicaid Services (CMS). [Accessed August 18, 2015] National Health Expenditure Projections: 2014-2024. 2015. Available at: https://www.cms.gov/Research-StatisticsData-and-Systems/Statistics-Trends-and-Reports/NationalHealthExpendData/Downloads/ proj2014.pdf

7. Burwell SM. Setting Value-Based Payment Goals - HHS Efforts to Improve U.S. Health Care. N Engl J Med. 2015; 372(10):897-899. [PubMed: 25622024]

8. Centers for Medicare \& Medicaid Services (CMS). Medicare Shared Savings Program: Accountable Care Organizations. Fed Regist. 2015; 80(110):32691-32845. [PubMed: 26065102]

9. Centers for Medicare \& Medicaid Services. [Accessed June 24, 2015] Shared Savings Program Accountable Care Organizations Provider-level RIF. Available at: http://www.resdac.org/cms-data/ files/ssp-aco-provider-level-rif

10. Centers for Medicare \& Medicaid Services. [Accessed June 24, 2015] Coding Specialty Codes. Available at: https://www.cms.gov/Research-Statistics-Data-and-Systems/Statistics-Trends-andReports/MedicareFeeforSvcPartsAB/downloads/SpecialtyCodes2207.pdf

11. Centers for Medicare \& Medicaid Services. [Accessed May 1, 2013] NPPES Downloadable File. Available at: http://download.cms.gov/nppes/NPI_Files.html

12. Bindman AB. Using the National Provider Identifier for Health Care Workforce Evaluation. MMRR. 2013; 3(3):1-10.

13. Centers for Medicare \& Medicaid Services. [Accessed June 26, 2015] Crosswalk: Medicare Provider/Supplier to Healthcare Provider Taxonomy. Available at: http://download.cms.gov/nppes/ NPI_Files.html

14. Centers for Medicare \& Medicaid Services. [Accessed February 1, 2015] Shared Savings Program Accountable Care Organizations (ACO) PUF. Available at: http://www.cms.gov/ResearchStatistics-Data-and-Systems/Downloadable-Public-Use-Files/SSPACO/Overview.html

15. SAS Institute. [Accessed August 18, 2015] Sample 24900: Label states on a US map with PROC GMAP. Available at: http://support.sas.com/kb/24/900.html

16. Dupree JM, Patel K, Singer SJ, et al. Attention to surgeons and surgical care is largely missing from early medicare accountable care organizations. Health Aff. 2014; 33:972-979.

17. Suskind AM, Clemens JQ. Affordable care act: Implications in female pelvic medicine and reconstructive surgery. Curr Urol Rep. 2014; 15:1-5.

18. Shortell S. A Taxonomy of Accountable Care Organizations for Policy and Practice. Health Serv Res. 2014; 46(6):1883-1899. [PubMed: 25251146]

19. American Urological Association. [Accessed on July 8, 2015] The State of Urology Workforce and Practice in the United States 2014. Available at: https://www.auanet.org/common/pdf/research/ census/State-Urology-Workforce-Practice-US.pdf

20. Centers for Medicare \& Medicaid Services. [Accessed August 17, 2015] Fast Facts: All Medicare Shared Savings Program ACOs. Available at https://www.cms.gov/Medicare/Medicare-Fee-forService-Payment/sharedsavingsprogram/Downloads/All-Starts-MSSP-ACO.pdf

21. McWilliams JM, Chernew ME, Dalton JB, Landon BE. Outpatient Care Patterns and Organizational Accountability in Medicare. JAMA Intern Med. 2014; 02115(6):1-8. 
22. Greenberg JO, Barnett ML, Spinks Ma, Dudley JC, Frolkis JP. The "medical neighborhood": integrating primary and specialty care for ambulatory patients. JAMA Intern Med. 2014; 174:454457. [PubMed: 24474526] 


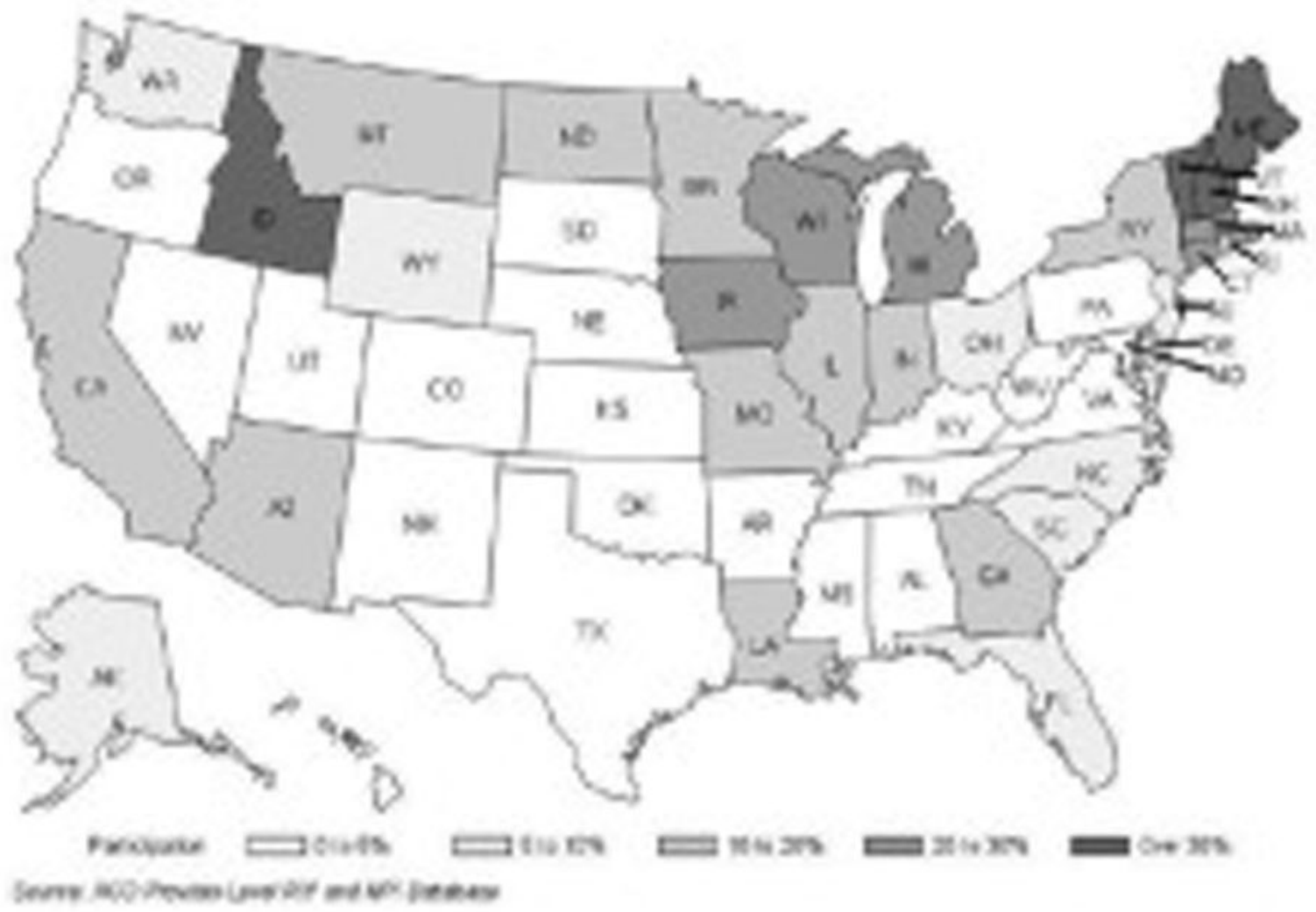

Figure 1.

Proportion of all practicing urologists in each of the 50 U.S. states that are formal participants in the MSSP ACO program. Puerto Rico is not included in this map. ${ }^{11}$ 


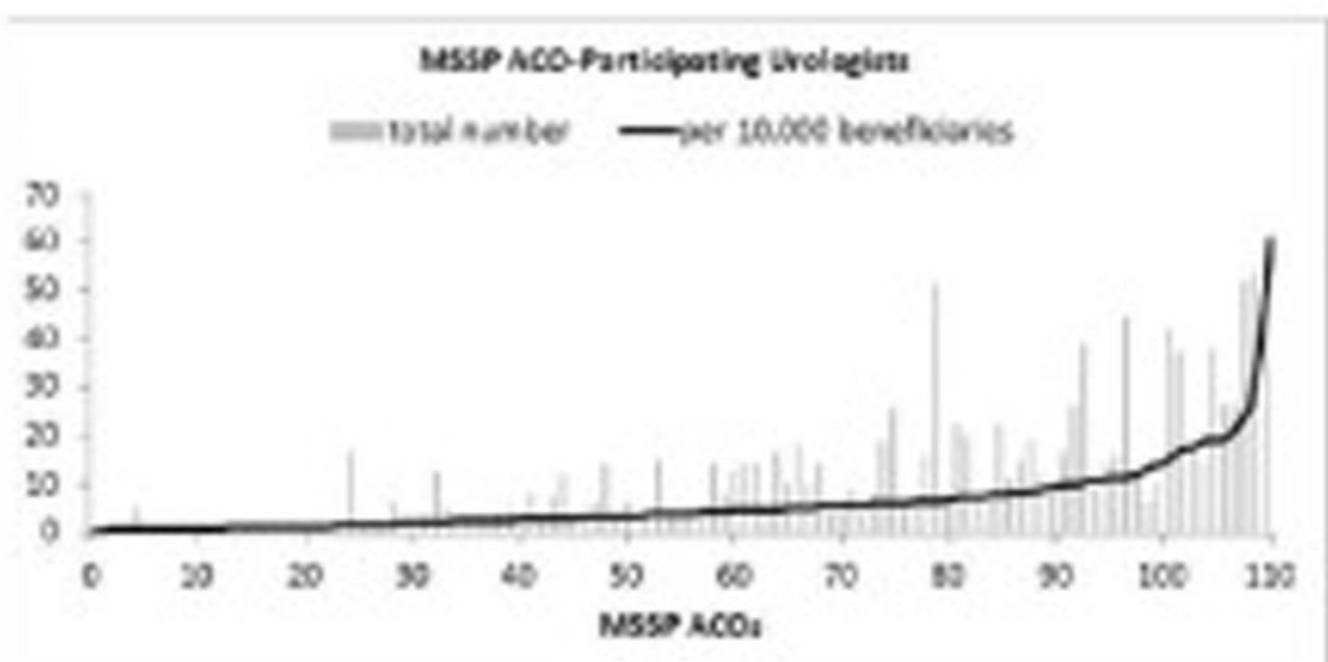

Figure 2.

Variation in urologist participation across 110 MSSP ACOs. The 110 MSSP ACOs not shown had no ACO-participating urologists. 
Table 1

Characteristics of urologists participating in MSSP ACOs.

\begin{tabular}{|c|c|c|c|}
\hline \multirow[b]{2}{*}{ Characteristic } & ACO-Participants & Non-Participants & \multirow[b]{2}{*}{ p-value } \\
\hline & $\mathrm{n}(\%)$ & n $(\%)$ & \\
\hline Total Urologists & 1,118 & 9,966 & \\
\hline \multicolumn{4}{|c|}{ U.S Census Region * } \\
\hline Northeast & $315(28)$ & $2,032(20)$ & \\
\hline Midwest & $387(35)$ & $2,045(21)$ & \\
\hline South & $201(18)$ & 3,904 (39) & $<0.001$ \\
\hline West & 215 (19) & $1,985(20)$ & \\
\hline \multicolumn{4}{|l|}{ Gender } \\
\hline Female & $117(10)$ & $785(8)$ & 0.003 \\
\hline
\end{tabular}

Puerto Rico included with states in the "South" U.S. Census Region. 
Table 2

Characteristics of patients and providers in MSSP ACOs by urologist participation

\begin{tabular}{lccc} 
Characteristics & ACOs with Urologists & ACOs without Urologists & p-value \\
\hline Total number of MSSP ACOs (\%) & $110(50)$ & $110(50)$ \\
\cline { 2 - 3 } & \multicolumn{2}{c}{ Mean } & $<0.001$ \\
\cline { 2 - 3 } Number of beneficiaries & 19,298 & 10,599 & $<0.001$ \\
Number of PCPs per 1,000 beneficiaries & 11.4 & 7.0 & $<0.001$ \\
Number of specialists per 1,000 beneficiaries & 25.8 & 7.0 & $<0.001$ \\
Number of advance practice clinicians per 1,000 beneficiaries & 4.2 & 2.7 & 0.9 \\
Percent of beneficiaries age $\geq 75$ & $40.0 \%$ & $39.9 \%$ & 0.5 \\
Percent of female beneficiaries & $57.5 \%$ & $57.6 \%$ & 0.4 \\
Percent of non-white beneficiaries & $16.2 \%$ & $18.1 \%$ & 0.7 \\
Percent of beneficiaries with ESRD & $1.0 \%$ & $1.1 \%$ & 0.8 \\
Percent of disabled beneficiaries & $14.9 \%$ & $14.6 \%$ & 0.9 \\
Percent of dual-eligible beneficiaries & $8.6 \%$ & $8.7 \%$ & \\
\hline
\end{tabular}

MSSP ACOs: Medicare Shared Savings Program Accountable Care Organizations

PCP: Primary Care Physicians

ESRD: End Stage Renal Disease

Advance Practice Clinicians: Nurse Practitioners, Physician Assistants, Certified Nurse Specialists 